\title{
Computed Tomography for 4-Dimensional Angiography and Perfusion Imaging of the Prostate for Embolization Planning of Benign Prostatic Hyperplasia
}

\author{
Kobe, Adrian ; Puippe, Gilbert ; Klotz, Ernst ; Alkadhi, Hatem ; Pfammatter, Thomas
}

\begin{abstract}
OBJECTIVES The aim of this study was to evaluate the feasibility of a computed tomography (CT) protocol enabling the visualization of the prostatic artery (PA) before prostatic artery embolization (PAE) in benign prostatic hyperplasia, which provides quantitative perfusion information of the prostate gland. MATERIALS AND METHODS In this institutional review board-approved study, 22 consecutive patients (mean age, $67 \pm 7$ years) who were planned to undergo PAE underwent a dynamic CT scan of the pelvis (scan range, $22.4 \mathrm{~cm}$; cycle time, 1.5 seconds; scan time, 44 seconds; 25 scan cycles; $70 \mathrm{kVp}$; $100 \mathrm{mAs}$ ) after the administration of $70 \mathrm{~mL}$ of iodinated contrast media (flow rate, $6 \mathrm{~mL} / \mathrm{s} ; 10$ seconds' delay). Image postprocessing consisted of a spatiotemporal, frequency-depending multiband filtering technique with noise reduction, motion correction, resulting in (1) time-resolved, temporal maximum intensity projection (MIP) images from fusion of multiple arterial time points; (2) 4-dimensional (4D) CT angiography images after bone and calcium plaque removal; and (3) parametric perfusion maps of the prostate. Intraprocedural cone-beam CT was performed with a microcatheter in the PA. In both modalities, the contrast-to-noise ratio of the right internal iliac artery or the PA was calculated, respectively. Visibility of the PA was scored using a Likert scale (score $1=$ not seen, to score $4=$ intraprostatic PA branches seen). Quantitative perfusion analysis of the dynamic pelvic CT included calculation of the blood flow, blood volume, mean transit time, and flow extraction product. RESULTS The average volume CT dose index and dose length product of CT was $35.7 \pm 6.8$ mGy and $737.4 \pm$ $146.3 \mathrm{mGy} \cdot \mathrm{cm}$, respectively. Contrast-to-noise ratio of the pelvic vessels on temporal MIP images and cone-beam CT were $45 \pm 19$ and $69 \pm 27$, respectively $(\mathrm{P}<0.01)$. The mean visibility score of the $\mathrm{PA}$ was $3.6 \pm 0.6$ for $4 \mathrm{D}-\mathrm{CT}$ angiography and $3.97 \pm 0.2$ for cone-beam CT $(\mathrm{P}<0.001)$. The PA was visualized in $100 \%$ of 4D-CT angiography examinations, with one PA being visible only proximally. Prostate CT perfusion analysis showed blood flow, blood volume, mean transit time, and flow extraction product values of $27.9 \pm 12.5 \mathrm{~mL} / 100 \mathrm{~mL} / \mathrm{min}, 2.0 \pm 0.8 \mathrm{~mL} / 100 \mathrm{~mL}, 4.5 \pm 0.5$ second, and $12.6 \pm 5.4$ $\mathrm{mL} / 100 \mathrm{~mL} / \mathrm{min}$, respectively, for the whole prostate gland. About half the patients showed a pronounced difference between the lobes. CONCLUSIONS We introduced a CT protocol for PAE planning providing excellent visualization of the PA on temporal MIP images and 4D-CT angiography at a reasonable dose and low contrast volume. In addition, quantitative perfusion information is available, which might be useful for outcome prediction after embolization.
\end{abstract}

DOI: https://doi.org/10.1097/RLI.0000000000000582

Posted at the Zurich Open Repository and Archive, University of Zurich

ZORA URL: https://doi.org/10.5167/uzh-171751

Journal Article

Published Version 
Originally published at:

Kobe, Adrian; Puippe, Gilbert; Klotz, Ernst; Alkadhi, Hatem; Pfammatter, Thomas (2019). Computed Tomography for 4-Dimensional Angiography and Perfusion Imaging of the Prostate for Embolization Planning of Benign Prostatic Hyperplasia. Investigative Radiology, 54(10):661-668.

DOI: https://doi.org/10.1097/RLI.0000000000000582 


\title{
Computed Tomography for 4-Dimensional Angiography and Perfusion Imaging of the Prostate for Embolization Planning of Benign Prostatic Hyperplasia
}

\author{
Adrian Kobe, $M D$, * Gilbert Puippe, MD, * Ernst Klotz, PhD, $†$ \\ Hatem Alkadhi, MD, MPH, EBCR, FESER, * and Thomas Pfammatter, MD*
}

\begin{abstract}
Objectives: The aim of this study was to evaluate the feasibility of a computed tomography (CT) protocol enabling the visualization of the prostatic artery (PA) before prostatic artery embolization (PAE) in benign prostatic hyperplasia, which provides quantitative perfusion information of the prostate gland.

Materials and Methods: In this institutional review board-approved study, 22 consecutive patients (mean age, $67 \pm 7$ years) who were planned to undergo PAE underwent a dynamic CT scan of the pelvis (scan range, $22.4 \mathrm{~cm}$; cycle time, 1.5 seconds; scan time, 44 seconds; 25 scan cycles; $70 \mathrm{kVp} ; 100 \mathrm{mAs}$ ) after the administration of $70 \mathrm{~mL}$ of iodinated contrast media (flow rate, $6 \mathrm{~mL} / \mathrm{s} ; 10$ seconds' delay). Image postprocessing consisted of a spatiotemporal, frequency-depending multiband filtering technique with noise reduction, motion correction, resulting in (1) time-resolved, temporal maximum intensity projection (MIP) images from fusion of multiple arterial time points; (2) 4-dimensional (4D) CT angiography images after bone and calcium plaque removal; and (3) parametric perfusion maps of the prostate. Intraprocedural cone-beam CT was performed with a microcatheter in the PA. In both modalities, the contrast-to-noise ratio of the right internal iliac artery or the PA was calculated, respectively. Visibility of the PA was scored using a Likert scale (score $1=$ not seen, to score $4=$ intraprostatic PA branches seen). Quantitative perfusion analysis of the dynamic pelvic CT included calculation of the blood flow, blood volume, mean transit time, and flow extraction product.
\end{abstract}

Results: The average volume CT dose index and dose length product of CT was $35.7 \pm 6.8 \mathrm{mGy}$ and $737.4 \pm 146.3 \mathrm{mGy} \cdot \mathrm{cm}$, respectively. Contrast-to-noise ratio of the pelvic vessels on temporal MIP images and cone-beam CT were $45 \pm 19$ and $69 \pm 27$, respectively $(P<0.01)$. The mean visibility score of the PA was $3.6 \pm 0.6$ for $4 \mathrm{D}-\mathrm{CT}$ angiography and $3.97 \pm 0.2$ for cone-beam CT $(P<0.001)$. The PA was visualized in $100 \%$ of $4 \mathrm{D}-\mathrm{CT}$ angiography examinations, with one PA being visible only proximally. Prostate CT perfusion analysis showed blood flow, blood volume, mean transit time, and flow extraction product values of $27.9 \pm 12.5 \mathrm{~mL} / 100 \mathrm{~mL} / \mathrm{min}, 2.0 \pm 0.8 \mathrm{~mL} / 100 \mathrm{~mL}, 4.5 \pm 0.5$ second, and $12.6 \pm 5.4 \mathrm{~mL} / 100 \mathrm{~mL} / \mathrm{min}$, respectively, for the whole prostate gland. About half the patients showed a pronounced difference between the lobes.

Conclusions: We introduced a CT protocol for PAE planning providing excellent visualization of the PA on temporal MIP images and 4D-CT angiography at a reasonable dose and low contrast volume. In addition, quantitative perfusion information is available, which might be useful for outcome prediction after embolization.

Key Words: computed tomography, perfusion, angiography, prostate, embolization

(Invest Radiol 2019;00: 00-00)

Received for publication March 11, 2019; and accepted for publication, after revision, April 16, 2019

From the *Institute of Diagnostic and Interventional Radiology, University Hospital Zurich, Zurich, Switzerland; and $\uparrow$ Siemens Healthineers, Forcheim, Germany. Conflicts of interest and sources of funding: none declared.

Correspondence to: Hatem Alkadhi, MD, MPH, EBCR, FESER, Institute of Diagnostic and Interventional Radiology, University Hospital Zurich, Rämistrasse 100, CH-8091 Zurich, Switzerland. E-mail: hatem.alkadhi@usz.ch.

Copyright (C) 2019 Wolters Kluwer Health, Inc. All rights reserved.

ISSN: 0020-9996/19/0000-0000

DOI: $10.1097 /$ RLI.0000000000000582
B enign prostatic hyperplasia (BPH) is a common disease of the older man with, after conservative treatment failure, transurethral resection of the prostate being the criterion standard treatment. Nevertheless, selective prostatic artery embolization (PAE) emerges as a minimally invasive treatment alternative. A positive effect on BPH was first described by Mitchell et $\mathrm{al}^{1}$ in 4 patients undergoing selective PAE to control massive prostatic bleeding. Elective PAE for BPH was first described by DeMeritt et $\mathrm{al}^{2}$ in 2000 . Recent studies showed that PAE is an effective alternative in reducing lower urinary tract symptoms caused by BPH with a favorable safety profile. ${ }^{3-7}$ Supported by the United Kingdom Register of Prostate Embolization study, ${ }^{3}$ the National Institute for Health and Care Excellence in the United Kingdom approved PAE for treatment of BPH in their updated guidelines. ${ }^{8}$

Nevertheless, there is still uncertainty on which patients profit most from PAE and which patients should be offered other treatment strategies. There are few studies analyzing imaging and clinical patterns, enabling the prediction of the outcome after PAE such as preprocedural high prostate gland volume and extent of prostate gland ischemia observed in early postinterventional magnetic resonance imaging (MRI). ${ }^{9-12}$ As PAE is a technically challenging procedure due to the highly variable anatomy of the prostatic artery (PA) and considering the often advanced atherosclerosis in the elderly male population, knowledge of the exact anatomy and morphology of the pelvic arteries including the PA is crucial for safe PAE. ${ }^{13,14}$

The primary purpose of this study was to evaluate the visibility of the PA before PAE in patients with BPH using a novel computed tomography (CT) protocol that provides 4-dimensional (4D) CT angiography information. Using a dynamic instead of static $\mathrm{CT}$ angiography protocol is motivated by the following reasons: with low $\mathrm{kV}$ protocols, the radiation exposure has a similar magnitude; in dynamic scanning, every vessel can be visualized at local peak opacification removing timing issues and potentially allowing to save contrast media; the inherent availability of precontrast information at identical scan setting enables CT digital subtraction angiography (DSA) techniques for plaque and bone removal. This approach, which allows generating "timinginvariate" CT angiography data, has been successfully used for cerebral and peripheral CTA applications before. ${ }^{15,16}$ A secondary aim of this study was to check the feasibility of also extracting quantitative gland perfusion information from the same data, which might serve as a potential outcome predictor.

\section{MATERIALS AND METHODS}

\section{Patients}

In total, 22 consecutive patients planned to undergo PAE at our institution from January to November 2018 were included into the study. All patients were evaluated by board-certified urologists before CT and were diagnosed with conservative therapy-refractory $\mathrm{BPH}$ and the recommendation of deobstructive surgery. In all patients, a preinterventional blood draw with measurements of the prostate-specific antigen was performed. Furthermore, the International Prostate Symptom Score was 
assessed. The study was approved by the local ethics committee, and informed consent was obtained from all patients.

\section{Prostate Dynamic Computed Tomography}

In all patients, preinterventional dynamic CT of the pelvis was obtained for treatment planning. All scans were performed on a third-generation dual-source CT scanner (SOMATOM Force; Siemens Healthineers, Forchheim, Germany). Dynamic CT of the pelvis was performed after the sublingual administration of 2 sprays of nitroglycerin (Isoket; Schwarz Pharma, Monheim, Germany) using $70 \mathrm{~mL}$ of iopromide (Ultravist 370, $370 \mathrm{mg} \mathrm{I} / \mathrm{mL}$; Bayer Schering Pharma, Berlin, Germany) at $6 \mathrm{~mL} / \mathrm{s}$ and a 10 -second scan delay. Contrast media injection was followed by a $20-\mathrm{mL}$ saline chaser at $6 \mathrm{~mL} / \mathrm{s}$. Scan range was $22.5 \mathrm{~cm}$ using the adaptive 4D spiral mode starting in the craniocaudal direction. Twenty-five scan cycles at a cycle time of 1.5 seconds and a total scan time of 44 seconds were performed. Tube voltage and currenttime product were set to $70 \mathrm{kVp}$ and $100 \mathrm{mAs}$, respectively. The scan was acquired in a single breath-hold. If breath-hold was not possible for the entire scan, patients were asked to breath superficially until scan end. Images were reconstructed with a slice width of $1.5 \mathrm{~mm}$ and an increment of $1 \mathrm{~mm}$ resulting in around 5600 images per study.

For radiation dose estimation, the volume $\mathrm{CT}$ dose index $\left(\mathrm{CTDI}_{\mathrm{vol}}\right.$; $\mathrm{mGy}$ ) and dose length product (DLP; $\mathrm{mGy} \cdot \mathrm{cm}$ ) from the automatically generated dose protocols were taken. Effective dose was calculated using the ICRP 103 updated conversion coefficient for the pelvis $(\kappa=0.015 \mathrm{mSv} /[\mathrm{mGy} \cdot \mathrm{cm}]) .{ }^{17-19}$ Although the coefficient for male patients is actually considerably lower $(0.010),{ }^{18}$ we chose to use the most conservative sex-averaged value published.

Image postprocessing was conducted on a standard postprocessing client server system (syngo.via; Siemens Healthineers, Erlangen, Germany) using dedicated applications (CT Dynamic Angio and CT Body Perfusion). Image postprocessing included noise reduction and automatic motion correction with generation of the following 3 image sets out of the single dynamic CT acquisition:

1. Temporal maximum intensity projection (MIP) images (syngo.via CT Dynamic Angio).

2. Four-dimensional CT angiography images including bone and calcium plaque removal (syngo.via Frontier Pelvic DSA). This step uses the temporal MIP data and precontrast baseline data simultaneously generated in step 1 .

3. Quantitative color-coded perfusion maps of blood flow (BF, $\mathrm{mL} /$ $100 \mathrm{~mL} / \mathrm{min})$, blood volume $(\mathrm{BV}, \mathrm{mL} / 100 \mathrm{~mL})$, mean transit time (MTT, seconds), and flow extraction product (FE, $\mathrm{mL} / 100 \mathrm{~mL} / \mathrm{min}$ ) from the same data (syngo.via CT Body Perfusion). Three-dimensional volumes of interest were placed around the whole prostate and around the right/left prostate lobe separately. Resulting quantitative perfusion parameters were recorded. In addition, the volume of interest around the whole prostate provided the prostate gland volume in milliliter.

\section{Prostate Artery Embolization-Cone-Beam CT}

Nineteen ( $86 \%$ ) of the 22 patients underwent PAE within 30 days after CT. Two patients decided to stay on medical therapy for BPH after initial workup with CT. One patient showed severe atherosclerotic changes and elongation of the pelvic arteries on temporal MIP images and was consequently offered surgical treatment.

Prostate artery embolization was performed in the 19 patients by the same interventional radiologist ( $>15$ years of experience). All interventions took place in a fully equipped angiography suite (ARTIS zeego and ARTIS pheno; Siemens Healthineers, Erlangen, Germany). Prostate artery embolization was performed in a standardized manner with a single-sided femoral access with initial DSA with the catheter placed in the internal iliac artery (40-degree ipsilateral oblique view) Subsequently, the PA was superselectively catheterized with placement of the microcatheter as distally as possible. For confirmation of correct microcatheter placement and identification of possible collaterals to other arteries, a cone-beam CT was performed by hand injection of 1 to $2 \mathrm{~mL}$ of $100 \%$ iodinated contrast media (Visipaque; GE Healthcare, Princeton, NJ). Image reconstruction was performed with a syngo $\mathrm{X}$ workplace (Siemens Healthineers, Erlangen, Germany).

For radiation dose estimation, the dose area product (DAP; $\mathrm{Gy} \cdot \mathrm{cm}^{2}$ ) and fluoroscopy time were documented. The total DAP of the PAE was documented as well as the DAP for fluoroscopy alone and cone-beam CT together with DSA. Effective dose was calculated using a standard conversion coefficient $\left(\kappa=0.26 \mathrm{mSv} / \mathrm{Gy}^{\circ} \mathrm{cm}^{2}\right)$.

\section{Image Quality}

Quantitative image quality was assessed in temporal MIP images and in cone-beam CT by calculating the contrast-to-noise ratio (CNR). To do so, a region of interest was placed in the proximal right internal iliac artery and in the right iliac muscle in temporal MIP images. In intraprocedural cone-beam CT, a region of interest was placed in the PA of both sides and in the obturator muscle on the ipsilateral side, as the iliac muscle was usually not in the field of view of cone-beam CT. For cone-beam CT, the mean CNR of the right and left side was used. The CNR was calculated for both modalities as follows:

Temporal MIP images: $\mathrm{CNR}=$ [attenuation (in HU) in the internal iliac artery - attenuation (in HU) in the iliac muscle $] \div$ standard deviation of attenuation in the iliac muscle.

Cone-beam CT: $\mathrm{CNR}=$ [attenuation (in HU) in the PA - attenuation (in $\mathrm{HU}$ ) in the obturator muscle $] \div$ standard deviation of attenuation in the obturator muscle.

Qualitative image quality was assessed using a 4-point Likert scale rating the visibility of the prostate artery for each pelvic side separately $(1=$ PA not visible, $2=$ PA only proximally visible; $3=$ PA visible in its complete course to the prostate without intraparenchymal branches; 4 = PA visible with intraparenchymal branches). A visibility score of 2 or higher was defined as being diagnostic. With a visibility score of 2, the iliac arteries are depicted sufficiently to identify exclusion criteria for PAE. In addition, the origin of the PA is identified that simplifies superselective catheterization during PAE. The PA was assessed in 4D-CT angiography after bone removal and cone-beam CT using the same criteria. Prostatic artery anatomy was classified in 4D-CT angiography according to de Assis et al. ${ }^{20}$

\section{Statistical Analysis}

For descriptive data, mean values and standard deviations are provided. Continuous variables were compared using the independent 2 -sample $\mathrm{t}$ test or paired $\mathrm{t}$ test, respectively. Categorical variables were compared using the $\chi^{2}$ test. All statistical analyses were performed with commercially available software (SPSS Statistics v. 23; IBM, Chicago, IL). Statistical significance was defined as a 2-tailed $P$ value below 0.05 .

\section{RESULTS}

Twenty-two patients (44 pelvic sides) were included in the study. Their mean age was $67 \pm 7$ years, the mean body mass index was $25.7 \pm 3.1 \mathrm{~kg} / \mathrm{m}^{2}$, the mean prostate volume was $87 \mathrm{~mL}$ (range, 41-196 mL), the mean prostate-specific antigen was $3.6 \pm 2.3 \mathrm{ng} / \mathrm{mL}$, and the mean International Prostate Symptom Score was 22.8 (range, 9-34; Table 1).

\section{Radiation Doses}

The average $\mathrm{CTDI}_{\mathrm{vol}}$, DLP, and effective dose of CT were $36.6 \pm 7.9 \mathrm{mGy}, 756.6 \pm 168.8 \mathrm{mGy} \cdot \mathrm{cm}$, and $11.3 \pm 2.5 \mathrm{mSv}$, respectively. 


\section{TABLE 1. Baseline Characteristics}

\begin{tabular}{lc}
\hline Patients, $\mathrm{n}$ & 22 \\
Age, mean $\pm \mathrm{SD}, \mathrm{y}$ & $67 \pm 6.6$ \\
$\mathrm{BMI}$, mean $\pm \mathrm{SD}$ (range), $\mathrm{kg} / \mathrm{m}^{2}$ & $25.7 \pm 3.1(18-33)$ \\
$\mathrm{IPSS}$ (range) & $22.8(9-34)$ \\
PSA, mean $\pm \mathrm{SD}, \mathrm{ng} / \mathrm{mL}$ & $3.6 \pm 2.3$ \\
Prostate volume (range), $\mathrm{mL}$ & $86.9(41-196)$
\end{tabular}

BMI indicates body mass index; IPSS, International Prostate Symptom Score; PSA, prostate-specific antigen.

The average total DAP, DAP (only fluoroscopy), DAP (DSA + conebeam $\mathrm{CT}$ ), and the effective dose of the PAE procedure were $415.4 \pm 208.1 \mathrm{~Gy} \cdot \mathrm{cm}^{2}, 105 \pm 67.6 \mathrm{~Gy} \cdot \mathrm{cm}^{2}, 310.5 \pm 148.7 \mathrm{~Gy} \cdot \mathrm{cm}^{2}$, and $108 \pm 54.1 \mathrm{mSv}$, respectively. The mean overall fluoroscopy time was $33.2 \pm 15.1$ minutes (Table 2 ). Seventy-six percent of the overall radiation dose was attributed to DSA and cone-beam CT.

\section{Image Quality and Anatomy}

According to de Assis et al, ${ }^{20}$ type I anatomy of the PA was present in $34.1 \%$, type II in $22.7 \%$, type III in $6.8 \%$, type IV in $31.8 \%$, and type $\mathrm{V}$ in $4.6 \%$.

The CNR in pelvic arteries was significantly higher in cone-beam CT $(69.1 \pm 26.9)$ than on temporal MIP images $(44.8 \pm 19, P<0.01)$.

The mean visibility score of the PA in 4D-CT angiography images was $3.6 \pm 0.6$. A visibility score of 4 was achieved in $57 \%$ of PA. In $41 \%$ of cases, a score of 3 was achieved. All 4D-CT angiography images were diagnostic with one PA being identified only proximally (visibility score 2) due to unilateral hip prosthesis. In the same patient, cone-beam CT had a visibility score of 3 due to beam-hardening artifacts. All other PA were scored with a visibility score of 4 (mean value, $3.97 \pm 0.2)$ in cone-beam CT, being significantly higher than in 4D-CT angiography images $(P<0.001$; Table 2$)$. Figure 1 shows 2 scans with a visibility score of 4 in 4D-CT angiography, temporal MIP images, and cone-beam CT. Figure 2 and Figure 3 illustrate intraprocedural DSA, 4D-CT angiography, and 3-dimensional cinematic rendering images.
In 2 pelvic sides (4.5\%), collaterals of the PA to the penile dorsal artery and superior vesical artery were seen on cone-beam CT needing superselective catheterization and microcoil placement to avoid nontarget embolization. Only the collateral to the superior vesical artery was also detectable on 4D-CT angiography. Furthermore, 2 pelvic sides showed tiny collaterals to the rectal artery with no change in treatment strategy. These collaterals were only seen in intraprocedural cone-beam $\mathrm{CT}$ and were not detectable on 4D-CT angiography even in retrospect.

\section{CT Perfusion Analysis}

In all but 1 patient (with unilateral hip prosthesis), quantitative $\mathrm{CT}$ perfusion analysis was feasible. The mean values for $\mathrm{BF}, \mathrm{BV}$, MTT, and FE product were $27.9 \pm 12.5 \mathrm{~mL} / 100 \mathrm{~mL} / \mathrm{min}$, $2.0 \pm 0.8 \mathrm{~mL} / 100 \mathrm{~mL}, 4.5 \pm 0.5 \mathrm{~second}$, and $12.6 \pm 5.4 \mathrm{~mL} / 100 \mathrm{~mL} /$ min, respectively. We frequently observed focal hyperintensities (Fig. 4). The distribution of the global mean values was approximately normally distributed (narrower for BF) with one outlier on the upper end. In $60 \%$ of the patients, the difference between the lobes was greater than $5 \%$; in $40 \%$ of the patients, it was greater than $10 \%$ (Table 3 and Fig. 5).

\section{DISCUSSION}

The present study introduces a novel dynamic CT protocol of the pelvis to determine the vascular anatomy including the PA and which provides additional quantitative information about the perfusion of the prostate gland.

Computed tomography angiography before PAE is performed in several institutions given its ability to depict pelvic vessels including variants and atherosclerotic changes, which is essential for treatment planning. ${ }^{13,14}$ Furthermore, patients with unfavorable anatomy can be excluded upfront and may be offered other treatment strategies. Tortuosity or stenosis of the pelvic arteries and its branches as well as excessive atherosclerosis are such exclusion criteria. Another reason to perform preprocedural CT angiography is radiation dose reduction during PAE. Mean fluoroscopy times for PAE range from 18 to 86 minutes in the literature. ${ }^{4,21}$ Both studies with the highest reported fluoroscopy times (55 and 86 minutes, respectively) did not perform preprocedural CT angiography of the pelvis. ${ }^{22,23}$ In contrast, the 2 studies with the lowest reported fluoroscopy times (18 and 20 minutes, respectively) performed CT angiography before PAE for anatomical assessment of the PA,

TABLE 2. Radiation Doses and Quantitative as well as Qualitative Image Assessments

\begin{tabular}{|c|c|c|c|}
\hline & 4D-CT Angiography & Intervention & $\boldsymbol{P}$ \\
\hline \multicolumn{4}{|l|}{ Radiation dose } \\
\hline $\mathrm{CTDI}_{\mathrm{vol}}, \mathrm{mGy}$ & $36.6 \pm 7.9$ & & \\
\hline DLP, $\mathrm{mGy} \cdot \mathrm{cm}$ & $756.6 \pm 168.8$ & & \\
\hline Total DAP, $\mathrm{Gy} \cdot \mathrm{cm}^{2}$ & & $415.4 \pm 208.1$ & \\
\hline DAP (fluoroscopy), Gy·cm ${ }^{2}$ & & $105 \pm 67.6$ & \\
\hline DAP (DSA + cone-beam CT), Gy $\mathrm{cm}^{2}$ & & $310.5 \pm 148.7$ & \\
\hline Fluoroscopy time, $\min$ & & $33.2 \pm 15.1$ & \\
\hline \multirow[t]{2}{*}{ Effective dose, $\mathrm{mSv}$} & $11.3 \pm 2.5^{*}$ & $108 \pm 54.1 \dagger$ & $<0.001$ \\
\hline & 4D-CT Angiography & Cone-Beam CT & \\
\hline \multicolumn{4}{|l|}{ Quantitative image assessment } \\
\hline Contrast-to-noise ratio & $44.8 \pm 19$ & $69.1 \pm 26.9$ & 0.002 \\
\hline \multicolumn{4}{|l|}{ Qualitative image assessment } \\
\hline PA visibility score & $3.6 \pm 0.6$ & $3.97 \pm 0.2$ & $<0.001$ \\
\hline Visibility of collaterals (needing coil embolization), $\%$ & 50 & 100 & \\
\hline $\begin{array}{l}{ }^{*} \text { Conversion coefficient }=0.015 \mathrm{mSv} /(\mathrm{mGy} \cdot \mathrm{cm}) . \\
\dagger \text { Conversion coefficient }=0.26 \mathrm{mSv} / \mathrm{Gy} \cdot \mathrm{cm}^{2} . \\
\text { CTDI }_{\text {vol }} \text { indicates volume CT dose index; DLP, dose lengt }\end{array}$ & ose area product; PA, pr & & \\
\hline
\end{tabular}



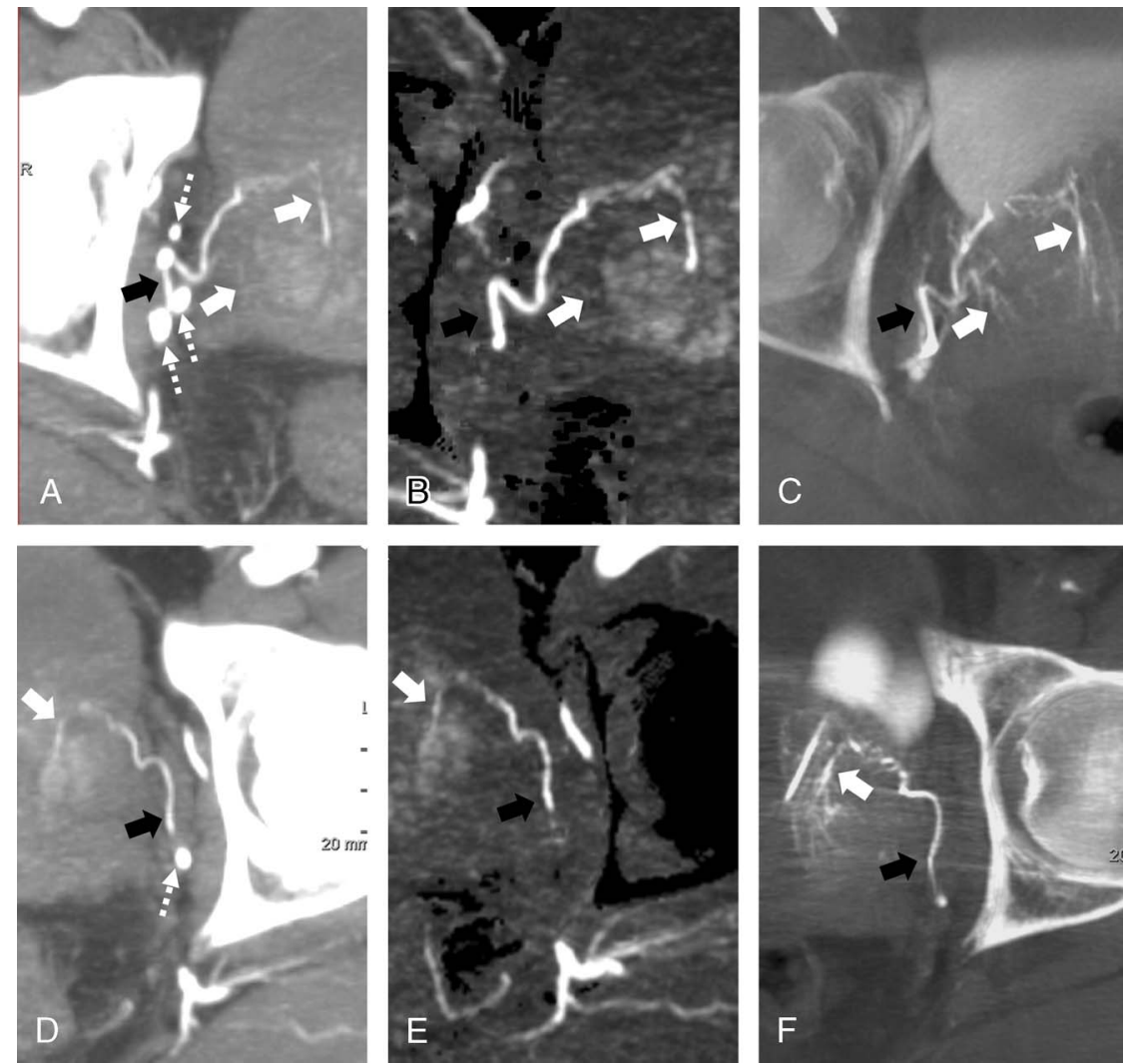

FIGURE 1. Preprocedural imaging of the prostate gland with the novel CT protocol compared with intraprocedural cone-beam CT. Panels A and D and panels $B$ and $E$ show the prostate artery (black arrows) with intraparenchymal branches (white arrows) in temporal MIP images and 4D-CT angiography in a 70-year-old patient with benign prostatic hyperplasia, respectively. Note the ability of bone and calcium removal in 4D-CT angiography (B and E) with complete removal of all phleboliths (dashed arrows) around the PA. Panels $\mathrm{C}$ and $\mathrm{F}$ show the corresponding artery (black arrows) and branches (white arrows) depicted by intraprocedural cone-beam CT.

which is most probably the result of faster superselective catheterization of the PA. ${ }^{24,25}$ The current study observed a mean fluoroscopy time of 33 minutes, which is at the lower published end.
Despite multiple scan cycles necessary (25 in total), the introduced CT protocol had a DLP of only $760 \mathrm{mGy} \cdot \mathrm{cm}$, which is at the lower range of previously published values from 800 to $1200 \mathrm{mGy} \cdot \mathrm{cm}$

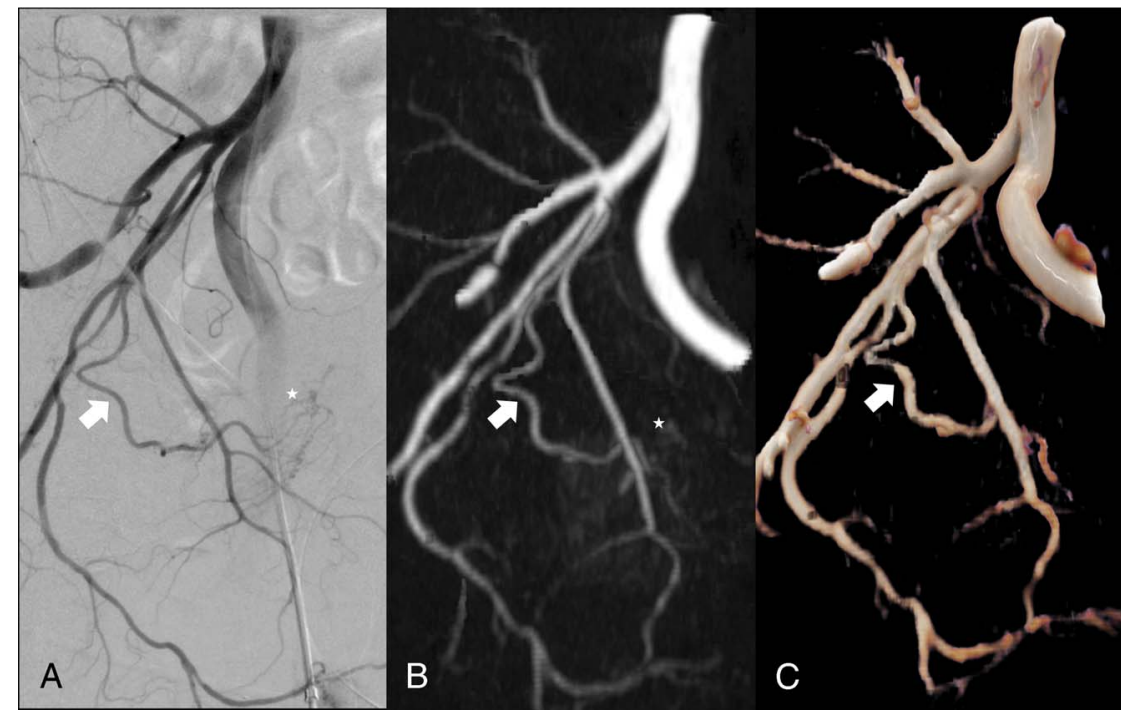

FIGURE 2. Intraprocedural digital subtraction angiography of the right pelvis (catheter placed in the internal iliac artery; ipsilateral oblique 40-degree view) depicting the right prostatic artery (white arrow; A). Four-dimensional CT angiography after bone subtraction (B) and 3D cinematic rendering image (C) of the right pelvic arterial anatomy with illustration of the prostatic artery (white arrow). Intraprostatic branches are seen with both modalities (white star). 

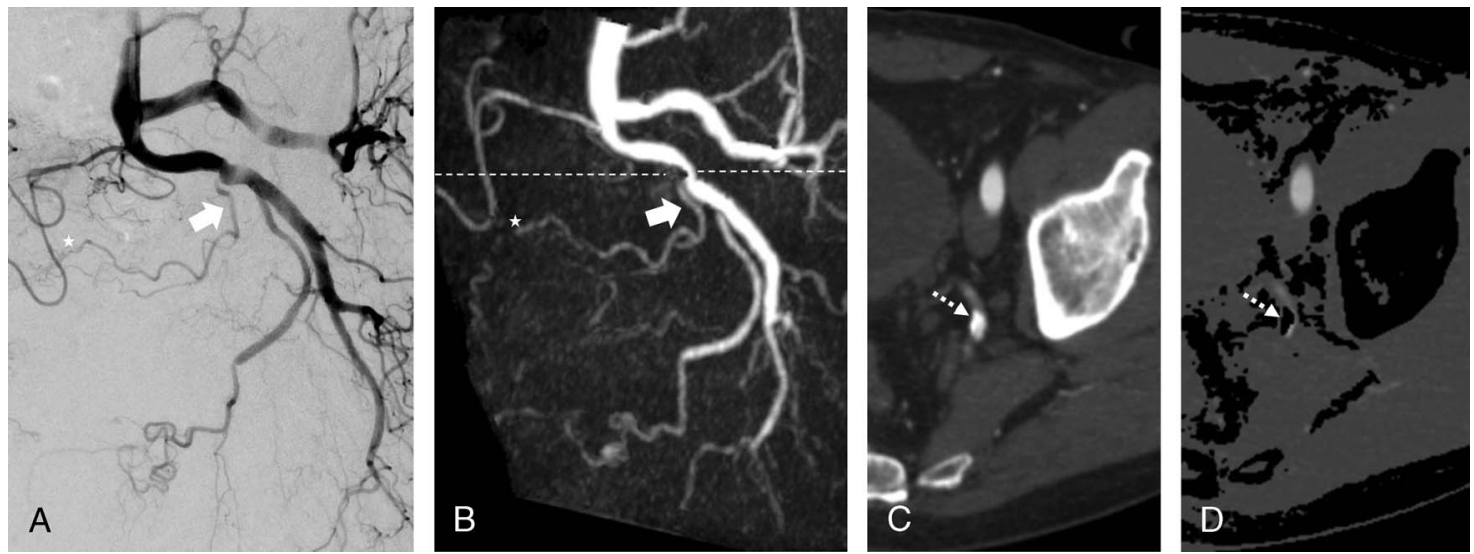

FIGURE 3. Intraprocedural digital subtraction angiography of the left pelvis (catheter placed in the internal iliac artery; ipsilateral oblique 40-degree view) depicting the left prostatic artery (white arrow; A). Four-dimensional CT angiography after bone subtraction (B) of the left pelvic arterial anatomy with illustration of the prostatic artery (white arrow). Intraprostatic branches are seen with both modalities (white star). Axial temporal MIP image (C) at the level of the dashed line depicted in panel B shows a calcium plaque (dashed arrow) in the internal iliac artery. Four-dimensional CT angiography image (D) shows removal of the calcium plaque (dashed arrow) after postprocessing enabled by the native baseline scan.

for conventional CT angiography of the pelvis. ${ }^{13,14,26}$ This is mainly due to the fact that the CT scanner used herein is able to acquire data with a low tube voltage of $70 \mathrm{kVp}$ (at a tube current-time product of $100 \mathrm{mAs}$ ). Low kilovoltage scanning goes along with low radiation doses, ${ }^{27}$ and additionally results in an increase in attenuation of contrast media due to scanning closer to the k-edge $(33.2 \mathrm{keV})$ of iodine ${ }^{28}$ Consequently, an increase in CNR is achieved by low tube voltage scanning. Despite the high natural attenuation of the hip joints, the CT protocol resulted in diagnostic images enabling the correct localization of the PA origin in all patients and sides, and depicted the complete course of the PA to the prostate in almost all (mean visibility score of 3.6). This excellent visualization of the pelvic arteries including the PA is the result of fusion of multiple arterial time points resulting in a single time-resolved, temporal MIP with low image noise. The latter is achieved by spatiotemporal, frequency-depending multiband filtering resulting in increased $\mathrm{CNR}$ at a lower radiation dose. In addition, in the pelvis, there is no issue with movement artifacts further improving image quality. Another advantage of this protocol with a total scan time of 44 seconds - compared with a single-phase CT angiography acquisition-is elimination of timing problems for ideal enhancement of the pelvic arteries. This might also explain that at similar dose values, only $70 \mathrm{~mL}$ of contrast media were required, this is about half of what has been used for standard $\mathrm{CT}$ angiography (120 to $150 \mathrm{~mL}){ }^{14,26}$ Furthermore, the native baseline scan enables optimal bone and calcium removal in image postprocessing resulting in 4D-CT angiography images with highly accurate depiction even of the distal, intraprostatic branches of the PA. So far, there is only

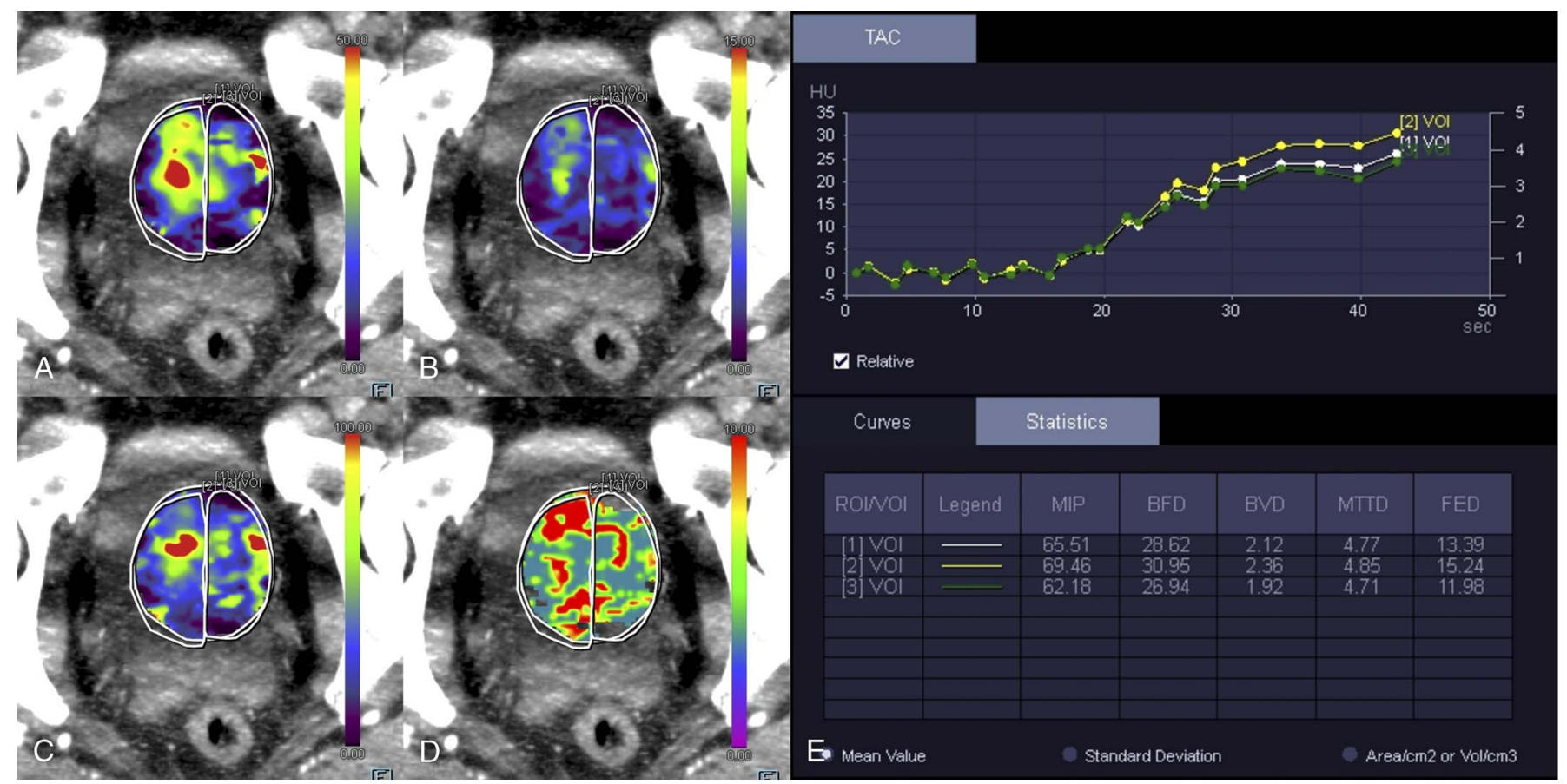

FIGURE 4. Postprocessed images of the prostate gland from perfusion CT. A volume of interest is placed around the entire prostate (VOI 1$)$ and another volume of interest is placed around the right and left prostatic lobe (VOI 2/VOI 3), respectively. Quantitative CT perfusion results are depicted in (E). Color-coded images of blood flow, blood volume, flow extraction product, and mean transit time are seen in panels A to D. 
TABLE 3. Prostate Perfusion Analysis

\begin{tabular}{lcc}
\hline & Whole Prostate & Lobe Difference (Range), \% \\
\hline Blood flow, $\mathrm{mL} / 100 \mathrm{~mL} / \mathrm{min}$ & $27.9 \pm 12.5$ & $11(1-33)$ \\
Blood volume, $\mathrm{mL} / 100 \mathrm{~mL}$ & $2.0 \pm 0.8$ & $11(0-33)$ \\
Flow extraction product, $\mathrm{mL} / 100 \mathrm{~mL} / \mathrm{min}$ & $12.6 \pm 5.4$ & $14(1-35)$ \\
Mean transit time, $\mathrm{s}$ & $4.5 \pm 0.5$ & $3(0-6)$ \\
\hline
\end{tabular}

one study in the literature analyzing the visibility of the PA with CT, reporting a visualization of intraparenchymal PA branches in 19\% of cases by single phase CT angiography. ${ }^{26}$ In contrast, with our CT protocol, we were able to visualize intraparenchymal branches in $57 \%$ of cases.

Vessel CNR in our study was still significantly higher in cone-beam CT compared with the proposed CT protocol due to direct superselective injection of contrast media with no dilution effects. But it is noteworthy, and also due to low $\mathrm{kV}$ usage, that $\mathrm{CNR}$ for intravenous injection was only $35 \%$ lower (45 vs 69 ) than for intra-arterial injection.

Intraprocedural cone-beam $\mathrm{CT}$ is considered crucial before PAE to avoid nontarget embolization due to collaterals to other arteries. Given the distal placement of the microcatheter in cone-beam CT, a mean visibility score of the PA of 3.97 was achieved, almost always depicting intraparenchymal branches and also visualizing collaterals of the PA. Collaterals of the PA to other arteries are described in $23 \%$ to $50 \%$ of PA. ${ }^{29-31}$ In our study, 2 PA showed collaterals needing coiling before embolization. Only one of these collaterals (50\%) was detected also in preprocedural CT, which is consistent with Maclean et $\mathrm{al}^{14}$ who found a sensitivity of collateral detection with CT angiography of only $59 \%$. Given the relatively low sensitivity of distal collateral detection in both conventional CT angiography and in the proposed dynamic pelvic CT protocol, it is still crucial to perform intraprocedural cone-beam CT for safe PAE.
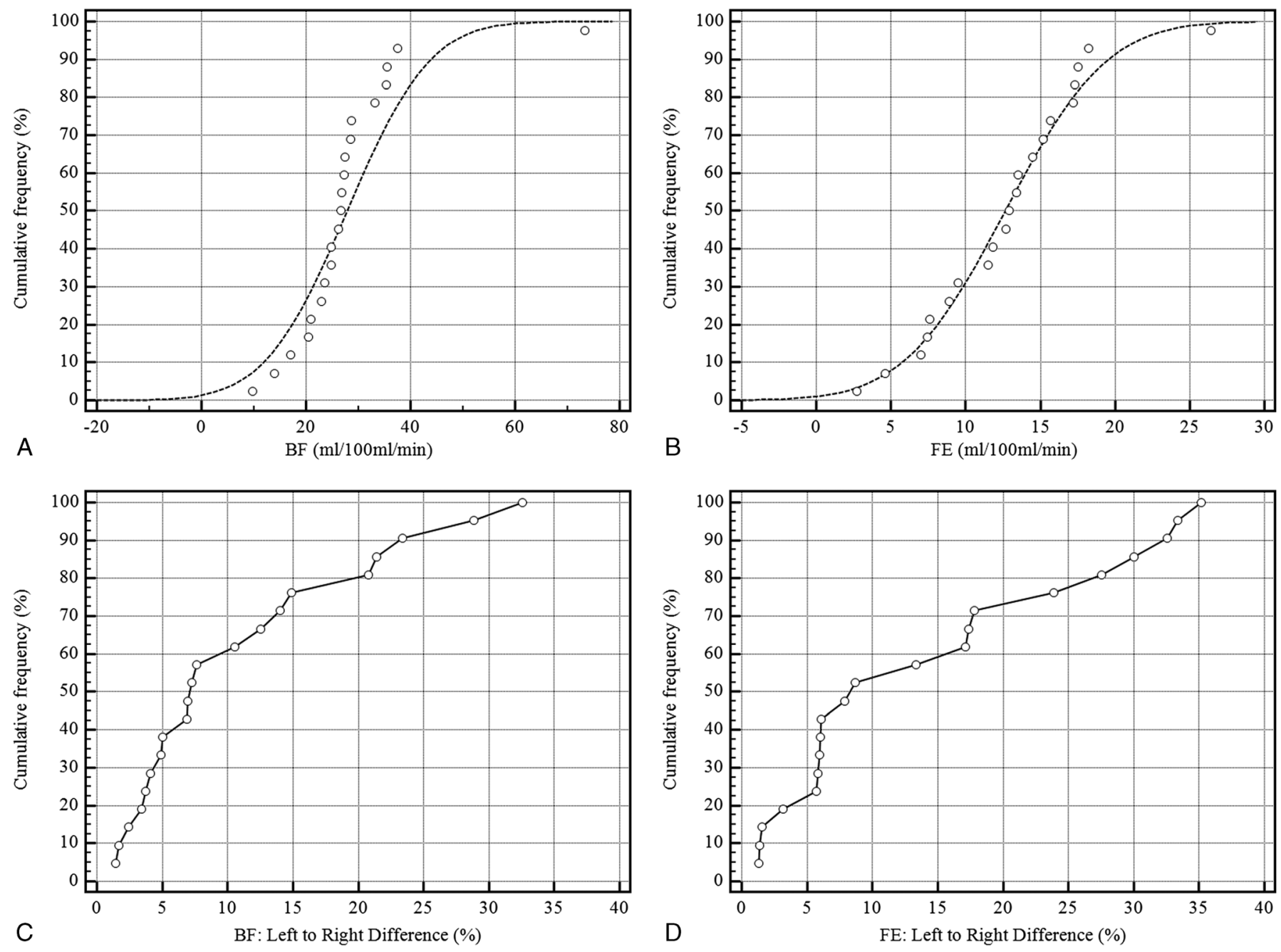

FIGURE 5. Distribution of blood flow (A) and flow extraction product (B) for the whole prostate. The dashed line is the corresponding normal distribution; there is one outlier on the upper end. Distribution of the absolute difference between left and right lobe for blood flow (C) and flow extraction product (D). Sixty percent of the patients have a difference greater than $5 \%, 40 \%$ greater than $10 \%$ between lobes. 
Despite its acknowledged efficacy in relieve of symptoms caused by BPH, there is still uncertainty on which patients are best candidates for PAE. There are studies showing that the initial prostate gland volume correlates with a favorable clinical outcome after PAE. ${ }^{9-11}$ Nevertheless, this was not confirmed by Bagla et $\mathrm{al}^{32}$ who found no differences in clinical outcome in patients stratified by preprocedural prostate gland volume. Other studies focused on early postinterventional parameters and found that the amount of prostate gland ischemia observed in early postinterventional MRI and prostate-specific antigen level 24 hours after intervention predicted clinical success of PAE. ${ }^{12,33}$

None of these studies took functional parameters such as perfusion data into account. One recent study by Brook et $\mathrm{al}^{34}$ studied the influence of embolic size on therapy response in a dog model. An increase of time to maximal perfusion intensity of the prostate right after PAE, assessed by dynamic contrast-enhanced MRI, showed a higher decrease in prostate volume. So far, only one study evaluated the vascularization of the prostate before embolization and found that increased vascularization was associated with favorable outcome after PAE. ${ }^{35}$ However, this study analyzed vascularization of the prostate only subjectively during intervention by DSA, which may suffer from subjectivity and limited reproducibility. Here, quantification of tissue perfusion with CT may be useful.

There is very limited literature on perfusion in $\mathrm{BPH}$, with most studies dealing with prostate cancer. ${ }^{36}$ Our injection protocol deviates slightly from typical oncological perfusion applications, because we injected for 12 seconds instead of typically not more than 10 seconds. ${ }^{37}$ This was done to achieve sufficient peak enhancement for CT angiography image generation. Still, we believe that this does not negatively influence perfusion results as, unlike cancer, normal and benign hyperplastic tissue does not have increased vascularity. Interestingly, there is one dynamic contrast-enhanced MRI study in BPH that reported plasma flow and $K_{\text {trans }}$ values $(\mathrm{mL} / 100 \mathrm{~mL} / \mathrm{min})$ of 21 and 10 , respectively, for the central gland and of 16 and 6 , respectively, for the peripheral zone. ${ }^{38}$ When converting our BF and FE values to plasma units (assuming a hematocrit level of 0.4 ), we obtain flow and $K_{\text {trans }}$ values of 17 and $8 \mathrm{~mL} /$ $100 \mathrm{~mL} / \mathrm{min}$, respectively, for the whole prostate. This is in very good agreement with the MRI study. ${ }^{38}$ We can only speculate if the frequently observed focal hyperintensities might be related to the nodular nature of $\mathrm{BPH}$, as we neither had a morphological reference nor outcome data. But as demonstrated in our study, perfusion analysis of the prostate seems feasible with CT providing quantitative information, which might be predictive for therapy response as shown in previous studies predicting the response to radioembolization in liver metastases ${ }^{39}$ and response to angiogenesis inhibitors in metastatic renal cell carcinoma, ${ }^{40}$ using perfusion CT performed before radio/chemotherapy.

We must acknowledge some study limitations. First, we included only a small sample size with 22 patients. Moreover, we have (so far) no outcome data available proving the usefulness of perfusion CT imaging as an outcome predictor for pretreatment workup. This needs to be investigated in future prospective trials. Another limitation of our protocol might be the image quality at a low tube voltage $(70 \mathrm{kVp})$ in obese patients. We did not encounter any problems with image quality at a fairly normal BMI distribution (mean, $25.7 \pm 3.1 \mathrm{~kg} / \mathrm{m}^{2}$ ) in this study. Use of higher tube voltages might overcome problems in obese patients, with the downside of resulting in higher radiation doses.

In conclusion, our study introduces a novel CT protocol of the pelvis enabling the visualization of the PA for treatment planning with temporal MIP images and 4D-CT angiography at a reasonable radiation dose and contrast volume. Whether the additional quantitative perfusion information of the prostate gland can serve as a potential outcome predictor after PAE requires further studies.

\section{REFERENCES}

1. Mitchell ME, Waltman AC, Athanasoulis CA, et al. Control of massive prostatic bleeding with angiographic techniques. J Urol. 1976;115:692-695.
2. DeMeritt JS, Elmasri FF, Esposito MP, et al. Relief of benign prostatic hyperplasia-related bladder outlet obstruction after transarterial polyvinyl alcohol prostate embolization. J Vasc Interv Radiol. 2000;11:767-770.

3. Ray AF, Powell J, Speakman MJ, et al. Efficacy and safety of prostate artery embolization for benign prostatic hyperplasia: an observational study and propensitymatched comparison with transurethral resection of the prostate (the UK-ROPE study). BJU Int. 2018;122:270-282.

4. Kuang M, Vu A, Athreya S. A Systematic Review of Prostatic Artery Embolization in the Treatment of Symptomatic Benign Prostatic Hyperplasia. Cardiovasc Intervent Radiol. 2016;7(suppl 7):S3-S9.

5. Uflacker A, Haskal ZJ, Bilhim T, et al. Meta-analysis of prostatic artery embolization for benign prostatic hyperplasia. J Vasc Interv Radiol. 2016;27:1686-1697.e8.

6. Cizman Z, Isaacson A, Burke C. Short- to midterm safety and efficacy of prostatic artery embolization: a systematic review. J Vasc Interv Radiol. 2016;27:1487-1493.e1.

7. Pisco JM, Bilhim T, Pinheiro LC, et al. Medium- and long-term outcome of prostate artery embolization for patients with benign prostatic hyperplasia: results in 630 patients. $J$ Vasc Interv Radiol. 2016;27:1115-1122.

8. NICE Guidance - Prostate artery embolisation for lower urinary tract symptoms caused by benign prostatic hyperplasia: C NICE (2018) Prostate artery embolisation for lower urinary tract symptoms caused by benign prostatic hyperplasia. BJU Int. 2018;122:11-12.

9. Abt D, Müllhaupt G, Mordasini L, et al. Outcome prediction of prostatic artery embolization: post hoc analysis of a randomized, open-label, non-inferiority trial. BJU Int. 2018.

10. Maclean D, Harris M, Drake T, et al. Factors predicting a good symptomatic outcome after prostate artery embolisation (PAE). Cardiovasc Intervent Radiol. 2018, 41:1152-1159.

11. de Assis AM, Maciel MS, Moreira AM, et al. Prostate zonal volumetry as a predictor of clinical outcomes for prostate artery embolization. Cardiovasc Intervent Radiol. 2017;40:245-251.

12. Bilhim T, Pisco J, Pereira JA, et al. Predictors of clinical outcome after prostate artery embolization with spherical and nonspherical polyvinyl alcohol particles in patients with benign prostatic hyperplasia. Radiology. 2016;281:289-300.

13. Bilhim T, Tinto HR, Fernandes L, et al. Radiological anatomy of prostatic arteries Tech Vasc Interv Radiol. 2012;15:276-285.

14. Maclean D, Maher B, Harris M, et al. Planning prostate artery embolisation: is it essential to perform a pre-procedural CTA? Cardiovasc Intervent Radiol. 2018; 41:628-632.

15. Smit EJ, Vonken EJ, van Seeters T, et al. Timing-invariant imaging of collateral vessels in acute ischemic stroke. Stroke. 2013;44:2194-2199.

16. Haubenreisser H, Bigdeli A, Meyer M, et al. From 3D to 4D: integration of temporal information into CT angiography studies. Eur J Radiol. 2015;84:2421-2424.

17. Huda W, Magill D, He W. CT effective dose per dose length product using ICRP 103 weighting factors. Med Phys. 2011;38:1261-1265.

18. Deak PD, Smal Y, Kalender WA. Multisection CT protocols: sex- and age-specific conversion factors used to determine effective dose from dose-length product. $R a$ diology. 2010;257:158-166.

19. Christner JA, Kofler JM, McCollough CH. Estimating effective dose for CT using dose-length product compared with using organ doses: consequences of adopting International Commission on Radiological Protection publication 103 or dual-energy scanning. AJR Am J Roentgenol. 2010;194:881-889.

20. de Assis AM, Moreira AM, de Paula Rodrigues VC, et al. Pelvic arterial anatomy relevant to prostatic artery embolisation and proposal for angiographic classification. Cardiovasc Intervent Radiol. 2015;38:855-861.

21. Jones P, Rai BP, Nair R, et al. Current status of prostate artery embolization for lower urinary tract symptoms: review of world literature. Urology. 2015;86:676-681.

22. de Assis AM, Moreira AM, de Paula Rodrigues VC, et al. Prostatic artery embolization for treatment of benign prostatic hyperplasia in patients with prostates $>$ 90 g: a prospective single-center study. J Vasc Interv Radiol. 2015;26:87-93.

23. Carnevale FC, da Motta-Leal-Filho JM, Antunes AA, et al. Quality of life and clinical symptom improvement support prostatic artery embolization for patients with acute urinary retention caused by benign prostatic hyperplasia. J Vasc Inter Radiol. 2013;24:535-542.

24. Pisco JM, Rio Tinto H, Campos Pinheiro L, et al. Embolisation of prostatic arteries as treatment of moderate to severe lower urinary symptoms (LUTS) secondary to benign hyperplasia: results of short- and mid-term follow-up. Eur Radiol. 2013 23:2561-2572.

25. Bilhim T, Pisco J, Campos Pinheiro L, et al. Does polyvinyl alcohol particle size change the outcome of prostatic arterial embolization for benign prostatic hyperplasia? Results from a single-center randomized prospective study. $J$ Vasc Interv Radiol. 2013;24:1595-602.e1.

26. Desai $\mathrm{H}, \mathrm{Yu} \mathrm{H}$, Ohana E, et al. Comparative analysis of cone-beam CT angiogram and conventional CT angiogram for prostatic artery identification prior to embolization. J Vasc Interv Radiol. 2018;29:229-232. 
27. Park EK, Seo BK, Kwon M, et al. Low-dose perfusion computed tomography for breast cancer to quantify tumor vascularity: correlation with prognostic biomarkers. Invest Radiol. 2019;54:273-281.

28. Higashigaito K, Husarik DB, Barthelmes J, et al. Computed tomography angiography of coronary artery bypass grafts: low contrast media volume protocols adapted to tube voltage. Invest Radiol. 2016;51:241-248.

29. Wang MQ, Duan F, Yuan K, et al. Benign prostatic hyperplasia: cone-beam CT in conjunction with DSA for identifying prostatic arterial anatomy. Radiology. 2017; 282:271-280.

30. Bagla S, Rholl KS, Sterling KM, et al. Utility of cone-beam CT imaging in prostatic artery embolization. J Vasc Interv Radiol. 2013;24:1603-1607.

31. Bilhim T, Pisco JM, Furtado A, et al. Prostatic arterial supply: demonstration by multirow detector angio CT and catheter angiography. Eur Radiol. 2011;21: 1119-1126.

32. Bagla S, Smirniotopoulos JB, Orlando JC, et al. Comparative analysis of prostate volume as a predictor of outcome in prostate artery embolization. $J$ Vasc Interv Radiol. 2015;26:1832-1838.

33. Kisilevzky N, Faintuch S. MRI assessment of prostatic ischaemia: best predictor of clinical success after prostatic artery embolisation for benign prostatic hyperplasia. Clin Radiol. 2016;71:876-882.
34. Brook OR, Faintuch S, Brook A, et al. Embolization therapy for benign prostatic hyperplasia: influence of embolization particle size on gland perfusion. J Magn Reson Imaging. 2013;38:380-387.

35. Gao YA, Huang Y, Zhang R, et al. Benign prostatic hyperplasia: prostatic arterial embolization versus transurethral resection of the prostate - a prospective, randomized, and controlled clinical trial. Radiology. 2014;270:920-928.

36. Huellner MW, Pauli C, Mattei A, et al. Assessment of prostate cancer with dynamic contrast-enhanced computed tomography using an en bloc approach. Invest Radiol. 2014;49:571-578.

37. Klotz E, Haberland U, Glatting G, et al. Technical Prerequisites and Imaging Protocols for CT Perfusion Imaging in Oncology. Eur J Radiol. 2015;2359-2367.

38. Kershaw LE, Hutchinson CE, Buckley DL. Benign prostatic hyperplasia: evaluation of T1, T2, and microvascular characteristics with T1-weighted dynamic contrast-enhanced MRI. J Magn Reson Imaging. 2009;29:641-648.

39. Morsbach F, Pfammatter T, Reiner CS, et al. Computed tomographic perfusion imaging for the prediction of response and survival to transarterial radioembolization of liver metastases. Invest Radiol. 2013;48:787-794.

40. Mains JR, Donskov F, Pedersen EM, et al. Dynamic contrast-enhanced computed tomography-derived blood volume and blood flow correlate with patient outcome in metastatic renal cell carcinoma. Invest Radiol. 2017;52:103-110. 